\title{
Impacto de la implantación de la norma ISO 9001:2000 en el Archivo General de la Universidad Complutense de Madrid
}

\author{
José Simón Martín*, Carlos Flores Varela**, Alicia Arias Coello***
}

Resumen: Este trabajo estudia el impacto que ha tenido la implantación de un sistema de gestión de la calidad, de acuerdo con los requisitos establecidos en la Norma Internacional ISO 9001:2000, en el Archivo General de la Universidad Complutense de Madrid. Para ello, se han analizado las opiniones del personal y de los usuarios del archivo antes y después de obtener la certificación de dicho sistema de gestión. Los resultados presentados señalan que la certificación no produce ninguna modificación en la percepción que tienen los usuarios del archivo sobre la calidad de los servicios de transferencia y búsqueda documental, así como sobre la calidad de la interacción entre el personal y los usuarios. En contraste, el personal valora muy favorablemente la certificación del archivo obteniéndose mejoras que son significativas en el área de gestión de los procesos de trabajo y, en concreto, en su documentación, coordinación entre procesos, definición de responsabilidades, o medición de su desempeño. Así mismo, y en opinión del personal, son significativas las mejoras que se producen con relación a algunos aspectos del funcionamiento del archivo como son: una mayor participación en el establecimiento de objetivos en su trabajo, una mayor identificación con la organización, y en la apreciación de que la mejora de la calidad del servicio es uno de los objetivos prioritarios que se deben conseguir. Estos resultados pueden servir como referencia para convencer a los directivos de otros servicios de información y de documentación, de las ventajas que tiene la implantación de un sistema de gestión de la calidad y su posterior certificación.

Palabras clave: Impacto certificación ISO, archivos e ISO 9001, ISO 9000 y servicios de información y documentación, calidad en los archivos.

\section{Impact of the introduction of the ISO 9001:2000 standard to the General Archive of the Complutense University of Madrid}

Abstract: This work studies the impact of the introduction of a quality management system, on the General. Archive of the Complutense University of Madrid in line with the requirements of International Standard ISO 9001:2000. For this study, the opinions of

* Instituto Universitario de Evaluación Sanitaria. Universidad Complutense de Madrid. Facultad de Medicina. Correo-e: iues@pdi.ucm.es.

** Archivo General de la Universidad Complutense de Madrid. Correo-e: caflores@pas.ucm.es.

**:* Departamento de Biblioteconomía y Documentación. Facultad de Ciencias de la Documentación. Universidad Complutense de Madrid. Correo-e: arias@pdi.ucm.es.

Recibido: 20-4-09; 2. ${ }^{a}$ versión: 29-5-09; aceptado: 1-6-09. 
the personnel and users of the archive before and after its obtaining certification with regard to the said management system were analysed. The results show that certification led to no change in user perception of the archive regarding the quality of document transfer and search services, or regarding the quality of interaction between personnel and users. In contrast, the personnel valued archive certification positively, regarding it as providing important improvements in the area of work process management, and specifically in its documentation process, the coordination between processes, the definition of responsibilities, and the measurement of performance. In the opinion of the archive personnel, the improvements made with respect to certain aspects of the functioning of the archive were significant, e.g., greater participation in establishing objectives, greater identification with organisation, and the appreciation that improvement of the quality of service should be a key objective. These results can be used as a reference tool to convince other information and documentation service managers of the advantages of introducing a quality management system and seeking subsequent certification.

Key words: Impact of ISO certification, archives and ISO 9001, ISO 9000 and information and documentation services, quality in archives.

\section{Introducción}

El propósito de este trabajo es presentar el impacto que ha tenido la implantación de un sistema de gestión de la calidad, de acuerdo con la Norma Internacional UNE-EN-ISO 9001:2000 en el Archivo General de la Universidad Complutense de Madrid (AGUCM). Este impacto ha sido medido teniendo en cuenta la opinión del personal y de los usuarios del archivo antes y después de conseguir la certificación de su sistema de gestión. Los aspectos que han sido objeto del estudio son en el caso del personal del archivo, la opinión sobre los recursos disponibles en el puesto de trabajo, la gestión de los procesos operativos del archivo, el liderazgo de la dirección, la organización de la unidad y la satisfacción con el trabajo. Los aspectos que se han valorado con respecto a los usuarios del archivo, han sido: la opinión sobre los procesos de transferencia y búsqueda documental, el trato recibido por el personal del archivo, las condiciones en que se desarrolla el trabajo en el archivo y la calidad del servicio.

En los últimos 20 años, los servicios de información y de documentación, entre los que se incluyen las bibliotecas, los archivos y los centros de documentación, han aplicado diferentes aproximaciones para mejorar la calidad de sus procesos con el fin de conseguir, la satisfacción de las necesidades y expectativas de sus clientes y de sus grupos de interés. Entre las metodologías más utilizadas por estas unidades de información está la implantación de sistemas de gestión de la calidad basados en la aplicación de los criterios del estándar internacional ISO 9001, con el que se certificaron 951.486 empresas en el año 2007 pertenecientes a 175 países. En España, el impacto de esta norma es tan grande que ocupa el cuarto lugar en número de certificaciones tras Japón, Italia y China. (The ISO Survey 2007. ISO Central Secretariat 2008. Switzerland).

En lo que se refiere a las bibliotecas, y a los servicios de información en general, la primera referencia que aparece en la literatura acerca de la implantación 
de un sistema de aseguramiento de la calidad se debe a Brophy (1994), que refiere su experiencia de certificación de la biblioteca de la Universidad Central de Lancashire. A partir de esta experiencia, un gran número de unidades de información han implantado un sistema de gestión de la calidad siguiendo la Norma Internacional ISO 9000.

Balagué Mola (2007) refiere en su tesis doctoral la existencia de 121 bibliotecas universitarias pertenecientes a 34 países, las cuales han certificado su sistema de gestión de la calidad, o están en fase de implantación de un sistema de este tipo. Por otro lado, Valls y Vergueiro (2006) incluyen en su artículo la experiencia de certificación, entre otras, de cinco bibliotecas no universitarias. Más recientemente Kostagiolas y Kitsiou (2008) informan de distintas experiencias de implantación de la Norma ISO 9001:2000 en las bibliotecas universitarias de Grecia.

En el caso de los centros de documentación, las referencias bibliográficas sobre experiencias en la implantación del estándar internacional ISO 9000 son escasas y en un análisis de las referencias que aparecen en la base de datos "Library and Information Science Abstracts" (LISA), nos encontramos con las siguientes: la Société Grenobloise d'Études et d'Applications Hydrauliques (Vasseur, 1998), el centro de documentación del Parc Natural de la Zona Volcánica de la Garrotxa (Grabolosa Sellabona y Vicens Perpinya, 2000), y el centro de información de patentes de Nuremberg (Krestel, 2006).

Respecto a los archivos, que es el objeto de este trabajo, la literatura publicada se centra, por un lado, en comentar el importante papel que tiene la gestión documental en la implantación y en el mantenimiento de un sistema de gestión de la calidad como el definido por los criterios de la Norma Internacional ISO 9000 (Lannon, 1995; Houten, 1996; Brumm, 1996; Stephens, 1996; Duff y McKemmish, 2000; Sebina, 2001 y Gunnlaugsdottir, 2002) y, por otro, en las amplias posibilidades que se abren a los profesionales de la gestión de la documentación, debido a la obligación que tienen las organizaciones que implantan este estándar, de desarrollar y mantener un sistema documental cuidadosamente controlado y gestionado (Weise y Stammoolis, 1993; Brumm, 1996; Yusof y Chell, 1998; Houten 1996, 2000 y Sebina, 2001).

Los ejemplos que nos encontramos en la literatura internacional sobre la implantación de la Norma ISO 9000:2000 en los archivos, son muy pocos, siendo de destacar el caso del Archivo Municipal de Oporto (Real, 2006). En España, las experiencias son más numerosas, perteneciendo la mayoría a los archivos y centros de documentación municipales: Departamento de Gestión Documental de la Diputación Provincial de Alicante (Martínez Mico, 2001), el Archivo Municipal de Logroño en 2002 (Murillo y López de Castro, 2002), el Archivo Municipal de Gijón en 2002 (Núñez Fernández, 2007), el Archivo Nacional de Cataluña en 2005 (Cruellas i Serra, Petit i Cibiriain, 2005), la Unidad de Sistemas de Información del Ayuntamiento de Zaragoza que incluye el archivo, hemeroteca y biblioteca y que fue certificado en 2007. Dentro de los archivos de universidades nos encontramos con los del Archivo de la Universidad Jaume I dependiente de la biblio- 
teca (Falomir y Segarra, 2006), o la certificación del Archivo General de la Universidad Complutense de Madrid en 2006 (Flores Varela, 2008).

El presente trabajo sigue la siguiente estructura: en la sección 2, en el apartado de métodos, se presenta el Archivo General de la Universidad Complutense y las características del estudio de opinión del personal y de los usuarios del AGUCM; en la sección 3, en el apartado de los resultados, se describe el impacto que ha tenido la certificación del AGUCM; y en la sección, 4 se discuten los resultados obtenidos y se presentan las conclusiones.

\section{Métodos}

\subsection{Presentación del AGUCM}

La Universidad Complutense de Madrid (UCM) fue creada en el siglo xv, y es una de las universidades más antiguas de Europa. Actualmente, la UCM tiene más de 80.000 alumnos, 6.000 profesores e investigadores, apoyados por más de 3.000 trabajadores de administración y servicios, distribuidos en dos campus que acogen a 26 centros docentes.

El Archivo General de la Universidad Complutense de Madrid tiene la misión de establecer y mantener un sistema de gestión integrada de los documentos que se generan en la universidad. El ciclo de gestión se inicia con la generación del documento en las unidades administrativas, a continuación se organizan los mismos y, finalmente, pasan a la etapa de conservación y custodia, o a la eliminación definitiva, de acuerdo con el marco legal existente. Al mismo tiempo, el AGUCM es la unidad responsable de facilitar a individuos, unidades administrativas y unidades docentes e investigadoras, el acceso al patrimonio documental de la UCM con el fin de promover el estudio y la difusión de la historia de la Universidad.

Inicialmente el AGUCM, solo controlaba la documentación más reciente producida por los servicios centrales de la universidad, mientras que el "Archivo Histórico" dependía de la Biblioteca. En 1999 se inició un proceso de reforma que empezó con el nombramiento de un archivero profesional como director del AGUCM y culminó con la integración, en el año 2006, del antiguo "Archivo Histórico". Desde entonces, el AGUCM integra todas las fases de la vida de los documentos de la universidad (Flores Varela 2003). Dado el gran volumen que ocupa esta documentación (más de 70.000 cajas), una parte importante de la misma ha sido confiada a una empresa de custodia externa.

En la actualidad, el AGUCM conforma una pequeña red de archivos que incluye dos archivos "centrales", los cuales recogen la documentación procedente de los servicios administrativos de la UCM, y un Archivo Intermedio e Histórico, donde se recoge la documentación procedente de los archivos centrales y de algunos centros docentes. Los documentos más antiguos se remontan a finales del siglo xv, y es de gran importancia el conjunto documental del siglo xIx, aun- 
que la mayor parte de la documentación está datada a partir de la década de 1980.

El equipo humano del AGUCM lo conforman un total de doce personas: seis técnicos, tres auxiliares de servicio y tres administrativos. Además, hay que añadir un número variable de becarios, entre tres y seis, reclutados de entre los estudiantes de últimos cursos de la Diplomatura de Biblioteconomía y Documentación y de las licenciaturas de Ciencias de la Documentación y de Historia.

\subsection{La estrategia de calidad en el AGUCM}

El AGUCM, inició su estrategia de calidad en el año 2002 (Flores Varela, 2008), participando, junto con otros servicios de la UCM, en una experiencia piloto de evaluación de la calidad en el marco del II Plan de Calidad de las Universidades (Ministerio de Educación, Cultura y Deporte, 2001).

Este proceso de evaluación comprendió dos etapas, una primera de autoevaluación del funcionamiento del AGUCM, siguiendo la Guía elaborada por el Consejo de Universidades y que se basaba en los criterios establecidos en el Modelo de Excelencia en la Gestión de la Fundación Europea para la Gestión de la Calidad (EFQM), y una segunda etapa de evaluación externa (Consejo de Universidades, 2000).

En mayo de 2004 se inició en el AGUCM el proyecto de implantación de un sistema de gestión de la calidad siguiendo el estándar UNE-EN-ISO 9001:2000. La dirección del proyecto recayó en la Oficina de Calidad y Desarrollo Estratégico de la UCM, organismo que realizó la asesoría para la implantación del sistema de gestión de la calidad y financió los costes del proyecto.

Como medida de apoyo al proceso de implantación del sistema de gestión, se formó al personal en el área de la gestión de la calidad y, en concreto, en la Norma UNE-EN-ISO 9001:2000; así mismo, cuatro personas del AGUCM recibieron formación adicional para convertirse en auditores internos.

El director del AGUCM, como responsable del sistema de gestión, identificó el mapa de procesos del archivo, que se representa en la Figura 1, con la participación del personal del archivo. A continuación se evaluó el funcionamiento de cada uno de estos procesos, y se elaboraron los procedimientos correspondientes y las instrucciones de trabajo, cuando fueron necesarias; así mismo se establecieron los criterios de aceptabilidad, los objetivos, los indicadores de rendimiento y las medidas para su control y monitorización.

Después de un período de tres meses de implantación del sistema de gestión de la calidad, el AGUCM solicitó la certificación a una entidad acreditada, la cual se consiguió en julio del 2006. 


\section{FIGURA 1}

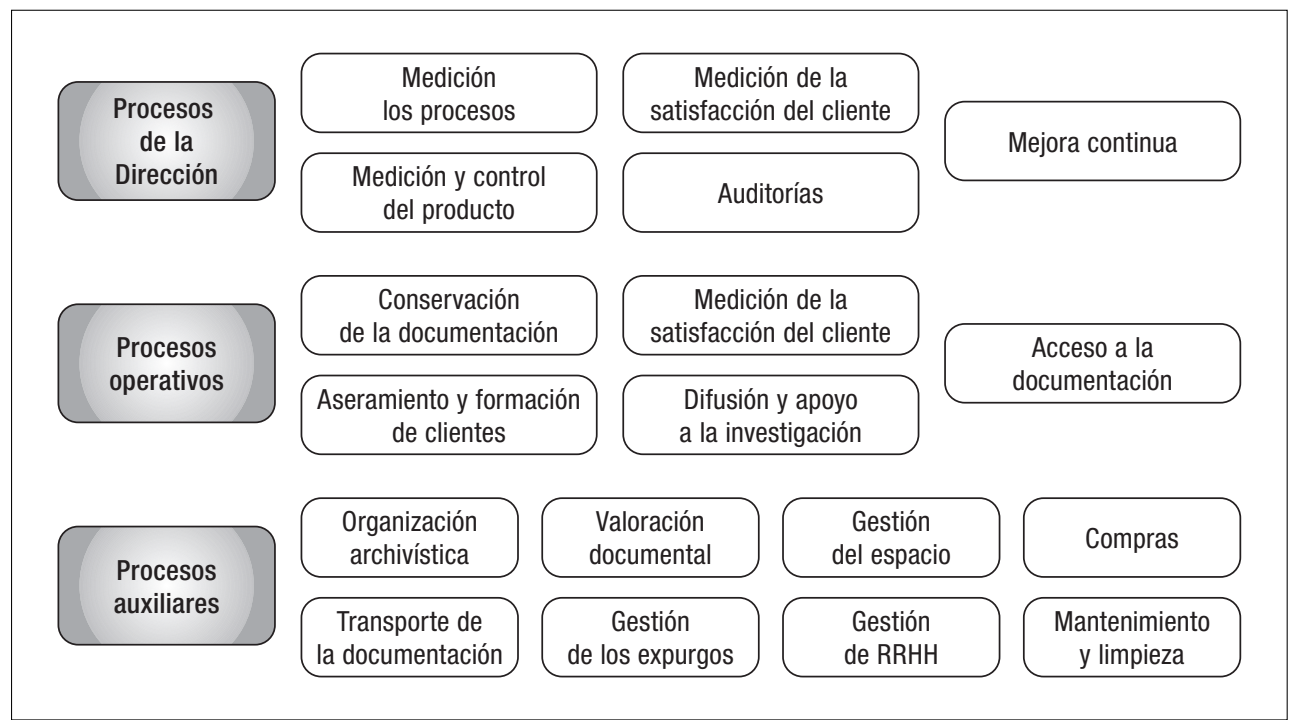

\subsubsection{Valoración del impacto de la implantación de un sistema de gestión de la calidad en el AGUCM}

La valoración del impacto de la implantación del sistema de gestión de la calidad, se ha realizado mediante el análisis de la opinión del personal del archivo y de los clientes del mismo, esto es, de las personas de otras unidades administrativas y de gestión de la UCM que se relacionan con el archivo. Estos estudios de opinión se han venido realizando desde el año 2002, de forma sistemática, cada dos años, fruto de una acción de mejora establecida en el proceso de evaluación del AGUCM anteriormente comentado. Los aspectos que han sido objeto de análisis proceden, en su mayor parte, de la Guía de Autoevaluación de Servicios realizada por el Consejo de Universidades en el año 2000.

Los aspectos presentados para su valoración a los usuarios del archivo, están orientados a conocer la satisfacción de los mismos con los procesos de transferencia y de búsqueda de documentos, la interacción con el personal del archivo, las condiciones de acceso al servicio y la valoración global que hacen sobre la calidad del servicio recibido; mientras que los aspectos presentados al personal del archivo, se relacionan con la calidad de los recursos, la capacidad de liderazgo de la dirección, el control y funcionamiento de los procesos operativos y el clima en el que se desarrolla el trabajo dentro del servicio.

Las preguntas de ambos tipos de cuestionarios disponen de una escala de valoración del 1 al 10, siendo el 1 la opinión más desfavorable y el 10 la más favorable. Los cuestionarios fueron enviados a cada una de las personas que utiliza el archivo (usuarios) y al personal que trabaja en el mismo. 
Para evaluar el impacto de la certificación, las opiniones del personal y la de los usuarios se dividieron en dos grupos. En un primer grupo se recogieron las opiniones emitidas antes de iniciarse el proceso de implantación del sistema de gestión de la calidad, y que se corresponden con las encuestas llevadas a cabo en los años 2002 y 2004. En un segundo grupo están las opiniones recogidas después de que se obtuviese la certificación del sistema de gestión de la calidad, y que se corresponden con los años 2006 y 2008. El número de cuestionarios recogidos en cada uno de ambos grupos es muy similar: en el caso del personal se analizaron 18 cuestionarios en el período previo a la certificación, y 20 en el período posterior, y en el caso de los usuarios del archivo, en el primer período se analizaron 46 cuestionarios, y en el segundo período 57.

En el estudio para cada pregunta se ha calculado el valor medio y el intervalo de confianza para una probabilidad del 95\%; así mismo para cada escala se ha llevado a cabo un análisis de varianza, seguido de un procedimiento de comparación múltiple de medias, para lo cual se ha verificado previamente la homogeneidad de varianzas de cada una de las muestras mediante la aplicación de los tests de Bartlett y de Levene y, posteriormente, se ha aplicado el Test de Bonferroni para comparación de medias dentro de cada una de las escalas utilizada (McClave, 1998).

\section{Resultados}

\subsection{Opinión del personal sobre el funcionamiento del AGUCM}

La opinión del personal se ha recogido sobre la siguiente categoría de cuestiones: recursos disponibles en el lugar de trabajo, liderazgo de la dirección, gestión de los procesos operativos, clima laboral y satisfacción general con el AGUCM.

Como se muestra en la Tabla I, la opinión que tiene el personal del AGUCM acerca de los recursos disponibles en el lugar del trabajo está por debajo del punto medio de la escala en el caso del espacio, el mobiliario, o las condiciones de seguridad e higiene. En el caso del equipamiento y aplicaciones informáticas que se utilizan, la opinión del personal es neutra. En estos casos, la certificación del sistema de gestión de la calidad, no modifica dichas opiniones del personal.

En la Tabla II se presenta la evolución de la opinión del personal del AGUCM sobre algunos aspectos relacionados con el funcionamiento de su unidad y el clima de trabajo existente. En todos los casos la opinión del personal mejora tras la implantación del sistema de gestión de la calidad. Sin embargo, esta mejora es significativa con un nivel de $\mathrm{p}<0,05$ en los aspectos relacionados con la participación del personal en la definición de los objetivos de la unidad, la opinión de que en los objetivos de su lugar de trabajo prima la mejora de la calidad del servicio y que el personal tiene un mayor sentimiento de ser parte integrante de un grupo de personas que trabajan con unos objetivos compartidos. 


\section{TABLA I}

\section{Opinión del personal del AGUCM sobre los recursos disponibles en el puesto de trabajo}

\begin{tabular}{|c|c|c|}
\hline Preguntas & $\begin{array}{l}\text { Años 2002-2004 } \\
\text { Valor medio (IC) }\end{array}$ & $\begin{array}{l}\text { Años 2006-2008 } \\
\text { Valor medio (IC) }\end{array}$ \\
\hline $\begin{array}{l}\text { R1. Dispongo del espacio adecuado para desarrollar co- } \\
\text { rrectamente mi trabajo. }\end{array}$ & $\begin{array}{c}4,67 \\
(3,52-5,81)\end{array}$ & $\begin{array}{c}4,65 \\
(3,44-5,86)\end{array}$ \\
\hline $\begin{array}{l}\text { R2. Dispongo del mobiliario adecuado para desarrollar } \\
\text { correctamente mi trabajo. }\end{array}$ & $\begin{array}{c}4,56 \\
(3,42-5,69)\end{array}$ & $\begin{array}{c}5,25 \\
(4,20-6,30)\end{array}$ \\
\hline $\begin{array}{l}\text { R3. Existen en mi lugar de trabajo las condiciones de se- } \\
\text { guridad e higiene apropiadas al mismo. }\end{array}$ & $\begin{array}{c}3,67 \\
(2,40-4,93)\end{array}$ & $\begin{array}{c}4,20 \\
(2,78-5,62)\end{array}$ \\
\hline $\begin{array}{l}\text { R4. Dispongo del equipamiento informático adecuado para } \\
\text { desarrollar correctamente mi trabajo. }\end{array}$ & $\begin{array}{c}5,22 \\
(4,08-6,36)\end{array}$ & $\begin{array}{c}6,95 \\
(5,70-8,20)\end{array}$ \\
\hline $\begin{array}{l}\text { R5. Las aplicaciones informáticas que utilizo están bien } \\
\text { adaptadas a mis necesidades. }\end{array}$ & $\begin{array}{c}5,11 \\
(4,24-6,00)\end{array}$ & $\begin{array}{c}7,00 \\
(5,80-8,20)\end{array}$ \\
\hline
\end{tabular}

(IC) Intervalo de confianza al 95,0\% de probabilidad.

Escala de 1 a 10 siendo el 1 la opinión más desfavorable y el 10 la más favorable.

\section{TABLA II}

Opinión del personal del AGUCM sobre aspectos relacionados con su unidad $y$ el clima en el trabajo

\begin{tabular}{|c|c|c|}
\hline Preguntas & $\begin{array}{l}\text { Años 2002-2004 } \\
\text { Valor medio (IC) }\end{array}$ & $\begin{array}{l}\text { Años 2006-2008 } \\
\text { Valor medio (IC) }\end{array}$ \\
\hline $\begin{array}{l}\text { CT1. Mi unidad dispone de un organigrama claro y bien } \\
\text { definido. }\end{array}$ & $\begin{array}{c}6,61 \\
(5,72-7,50)\end{array}$ & $\begin{array}{c}8,20 \\
(7,39-9,01)\end{array}$ \\
\hline $\begin{array}{l}\text { CT2. Las funciones y tareas asignadas a mi puesto de tra- } \\
\text { bajo están claramente definidas. }\end{array}$ & $\begin{array}{c}6,94 \\
(6,03-7,85)\end{array}$ & $\begin{array}{c}8,63 \\
(8,03-9,24)\end{array}$ \\
\hline $\begin{array}{l}\text { CT3. Las cargas de trabajo de las personas de mi unidad } \\
\text { están bien distribuidas. }\end{array}$ & $\begin{array}{c}5,67 \\
(4,80-6,54)\end{array}$ & $\begin{array}{c}8,00 \\
(7,26-8,74)\end{array}$ \\
\hline $\begin{array}{l}\text { CT4. He recibido información detallada sobre los objetivos } \\
\text { de mi lugar de trabajo. }\end{array}$ & $\begin{array}{c}5,78 \\
(4,69-6,86)\end{array}$ & $\begin{array}{c}7,30 \\
(6,02-8,58)\end{array}$ \\
\hline $\begin{array}{l}\text { CT5. He participado en la definición de los objetivos de } \\
\text { mi unidad. }\end{array}$ & $\begin{array}{c}3,94 \\
(2,47-5,42) \\
\end{array}$ & $\begin{array}{c}7,05^{* *} \\
(5,77-8,32) \\
\end{array}$ \\
\hline $\begin{array}{l}\text { CT6. En los objetivos de mi lugar de trabajo prima la me- } \\
\text { jora de la calidad de los servicios. }\end{array}$ & $\begin{array}{c}6,28 \\
(4,94-7,62) \\
\end{array}$ & $\begin{array}{c}8,55^{* *} \\
(7,74-9,36) \\
\end{array}$ \\
\hline $\begin{array}{l}\text { CT7. Me considero parte integrante de un grupo de per- } \\
\text { sonas que trabajo por tareas y objetivos compartidos. }\end{array}$ & $\begin{array}{c}6,00 \\
(4,96-7,04)\end{array}$ & $\begin{array}{c}8,65^{* *} \\
(7,81-9,48)\end{array}$ \\
\hline $\begin{array}{l}\text { CT8. El trabajo que realizo es acorde a los conocimientos } \\
\text { y experiencias que poseo. }\end{array}$ & $\begin{array}{c}7,22 \\
(6,59-7,85)\end{array}$ & $\begin{array}{c}8,05 \\
(7,06-9,04)\end{array}$ \\
\hline
\end{tabular}


TABLA II (CONTINUACIÓN)

\begin{tabular}{l|c|c}
\hline \multicolumn{1}{c|}{ Preguntas } & $\begin{array}{c}\text { Años 2002-2004 } \\
\text { Valor medio (IC) }\end{array}$ & $\begin{array}{c}\text { Años 2006-2008 } \\
\text { Valor medio (IC) }\end{array}$ \\
\hline $\begin{array}{l}\text { CT9. La formación que recibo es adecuada a mi puesto } \\
\text { de trabajo. }\end{array}$ & $\begin{array}{c}6,65 \\
(5,64-7,66)\end{array}$ & $\begin{array}{c}8,25 \\
(7,34-9,16)\end{array}$ \\
\hline $\begin{array}{l}\text { CT10. La comunicación interna en el servicio funciona } \\
\text { correctamente. }\end{array}$ & $\begin{array}{c}7,11 \\
(5,98-8,24)\end{array}$ & $\begin{array}{c}8,15 \\
(7,36-8,94)\end{array}$ \\
\hline CT11. Me satisface el trabajo que realizo. & 7,67 & 8,40 \\
& $(6,61-8,72)$ & $(7,35-9,44)$ \\
\hline CT12. Considero que el ambiente de trabajo es bueno. & 7,11 & 8,20 \\
& $(5,85-8,37)$ & $(7,10-9,30)$ \\
\hline CT13. Se fomenta el trabajo de equipo. & 7,35 & 8,10 \\
& $(6,18-8,53)$ & $(6,89-9,31)$ \\
\hline
\end{tabular}

(IC) Intervalo de confianza al 95,0\% de probabilidad.

** La diferencia es estadísticamente significativa a un nivel de 0,05.

Escala de 1 a 10 siendo el 1 la opinión más desfavorable y el 10 la más favorable.

En la Tabla III se presenta los resultados sobre la opinión que tiene el personal del AGUCM sobre sus directivos. Como se observa, el personal del Archivo tiene una opinión muy favorable sobre sus directivos. Esta opinión mejora en todos los aspectos tras la certificación del sistema de gestión de la calidad. Sin

\section{TABLA III}

Opinión del personal del AGUCM sobre aspectos relacionados con el liderazgo de la dirección

\begin{tabular}{|c|c|c|}
\hline Preguntas & $\begin{array}{l}\text { Años 2002-2004 } \\
\text { Valor medio (IC) }\end{array}$ & $\begin{array}{l}\text { Años 2006-2008 } \\
\text { Valor medio (IC) }\end{array}$ \\
\hline $\begin{array}{l}\text { L1. Puedo observar compromiso personal de mis jefes en } \\
\text { la mejora de la calidad. }\end{array}$ & $\begin{array}{c}6,61 \\
(5,63-7,59)\end{array}$ & $\begin{array}{c}8,75 \\
(7,96-9,54)\end{array}$ \\
\hline $\begin{array}{l}\text { L2. Mis jefes me ayudan aponer en marcha mis iniciativas } \\
\text { para mejorar el trabajo. }\end{array}$ & $\begin{array}{c}6,33 \\
(5,25-7,41)\end{array}$ & $\begin{array}{c}8,30 \\
(7,29-9,31)\end{array}$ \\
\hline $\begin{array}{l}\text { L3. Se me reconoce cuando introduzco una mejora en mi } \\
\text { trabajo. }\end{array}$ & $\begin{array}{c}5,67 \\
(4,15-7,18)\end{array}$ & $\begin{array}{c}8,35^{* *} \\
(7,34-9,36)\end{array}$ \\
\hline L4. Tengo fácil acceso a mis jefes. & $\begin{array}{c}7,90 \\
(6,82-8,95)\end{array}$ & $\begin{array}{c}8,60 \\
(7,37-9,83)\end{array}$ \\
\hline $\begin{array}{l}\text { L5. Ante contratiempos o dificultades me siento reforzado } \\
\text { y animado por mi superior. }\end{array}$ & $\begin{array}{c}6,39 \\
(5,04-7,73)\end{array}$ & $\begin{array}{c}8,40 \\
(7,60-9,19)\end{array}$ \\
\hline
\end{tabular}

(IC) Intervalo de confianza al 95,0\% de probabilidad.

* La diferencia es estadísticamente significativa a un nivel de 0,05.

Escala de 1 a 10 siendo el 1 la opinión más desfavorable y el 10 la más favorable. 
embargo, esta mejoría es solo significativa con respecto al reconocimiento que la dirección realiza cuando el personal introduce una mejora en su trabajo.

Los cambios más importantes que se producen en la opinión del personal después de implantarse el sistema de gestión de la calidad, ocurren con respecto a la forma en que se llevan a cabo los procesos en el AGUCM. Como se puede observar en la Tabla IV, es significativa la mejora en la apreciación que tiene el personal tras la certificación del sistema de gestión en cuanto a la documentación de los procesos, su medición, la identificación de los responsables, así como del nivel de coordinación entre los diferentes procesos que se realizan en el archivo.

\section{TABLA IV}

\section{Opinión del personal del AGUCM sobre aspectos relacionados con la gestión} de los procesos en el archivo

\begin{tabular}{l|c|c}
\hline \multicolumn{1}{c|}{ Preguntas } & $\begin{array}{c}\text { Años 2002-2004 } \\
\text { Valor medio (IC) }\end{array}$ & $\begin{array}{c}\text { Años 2006-2008 } \\
\text { Valor medio (IC) }\end{array}$ \\
\hline $\begin{array}{c}\text { P1. Los procesos que se gestionan en mi unidad están } \\
\text { documentados. }\end{array}$ & $\begin{array}{c}5,94 \\
(4,94-6,95)\end{array}$ & $\begin{array}{c}8,70^{* *} \\
(7,89-9,50)\end{array}$ \\
\hline $\begin{array}{l}\text { P2. Se miden periódicamente en la unidad indicadores de } \\
\text { funcionamiento de los procesos. }\end{array}$ & $\begin{array}{c}5,12 \\
(3,73-6,50)\end{array}$ & $\begin{array}{c}8,40^{* *} \\
(7,58-9,22)\end{array}$ \\
\hline $\begin{array}{l}\text { P3. Se produce una buena coordinación con otros servicios } \\
\text { que intervienen en los mismos. }\end{array}$ & $\begin{array}{c}6,39 \\
(5,66-7,11)\end{array}$ & $\begin{array}{c}7,85 \\
(7,09-8,61)\end{array}$ \\
\hline $\begin{array}{c}\text { P4. Los responsables de los procesos están identificados. } \\
(5,28-7,06)\end{array}$ & $\begin{array}{c}8,55^{* *} \\
(7,80-9,30)\end{array}$ \\
\hline P5. Se hacen reuniones para coordinar procesos entre \\
unidades.
\end{tabular}

(IC) Intervalo de confianza al 95,0\% de probabilidad.

** La diferencia es estadísticamente significativa a un nivel de 0,05 .

Escala de 1 a 10 siendo el 1 la opinión más desfavorable y el 10 la más favorable.

\subsection{Opinión de los usuarios del archivo}

Los usuarios del AGUCM tenían, en el período 2002-2004, una opinión muy favorable sobre la calidad global del servicio que se les presta, llegando a alcanzar ésta una valoración media de 7,89 sobre 10. Esta opinión se mantuvo estable durante el período 2006-2008 tras la implantación del sistema de gestión de la calidad, alcanzando un valor medio de 7,9.

La opinión que tienen los usuarios del AGUCM sobre la rapidez y sencillez con que se realiza el proceso de transferencias de documentos, desde las unidades administrativas al archivo, es muy favorable ya que alcanza valoraciones por encima del 7 (ver Tabla V). Estas opiniones no se modifican tras la certificación del sistema de gestión de la calidad. 
TABLA V

Opinión de los usuarios del AGUCM sobre el proceso de transferencia documental

\begin{tabular}{l|c|c}
\hline \multicolumn{1}{c|}{ Preguntas } & $\begin{array}{c}\text { Años 2002-2004 } \\
\text { Valor medio (IC) }\end{array}$ & $\begin{array}{c}\text { Años 2006-2008 } \\
\text { Valor medio (IC) }\end{array}$ \\
\hline TC1. Rapidez en el proceso de transferencia. & $\begin{array}{c}7,56 \\
(6,95-8,16)\end{array}$ & $\begin{array}{c}7,64 \\
(7,07-8,21)\end{array}$ \\
\hline TC2. Sencillez del proceso de transferencia. & $\begin{array}{c}7,45 \\
(6,82-8,09)\end{array}$ & $\begin{array}{c}7,11 \\
(6,54-7,67)\end{array}$ \\
\hline TC4. Utilidad del proceso de transferencia & 7,77 & 7,86 \\
& $(7,14-8,41)$ & $(7,34-8,38)$ \\
\hline
\end{tabular}

(IC) Intervalo de confianza al 95,0\% de probabilidad.

Escala de 1 a 10 siendo el 1 la opinión más desfavorable y el 10 la más favorable.

Los usuarios del AGUCM muestran también una opinión muy favorable con respecto a las características que reúne el personal del archivo (trato, profesionalidad, rapidez, información, etc.). Como se puede observar en la Tabla VI, esta opinión no se modifica tras la certificación del sistema de gestión de la calidad.

\section{TABLA VI}

Opinión de los usuarios del AGUCM sobre el trato recibido por el personal durante el proceso de búsquedas documentales

\begin{tabular}{l|c|c}
\hline \multicolumn{1}{c|}{ Preguntas } & $\begin{array}{c}\text { Años 2002-2004 } \\
\text { Valor medio (IC) }\end{array}$ & $\begin{array}{c}\text { Años 2006-2008 } \\
\text { Valor medio (IC) }\end{array}$ \\
\hline BD1. Le han tratado con amabilidad y consideración. & $\begin{array}{c}9,11 \\
(8,81-9,40)\end{array}$ & $\begin{array}{c}8,80 \\
(8,42-9,18)\end{array}$ \\
\hline BD2. Le han garantizado intimidad y confidencialidad. & $\begin{array}{c}8,643 \\
(8,17-9,12)\end{array}$ & $\begin{array}{c}8,48 \\
(8,03-8,93)\end{array}$ \\
\hline BD3. Le han atendido con rapidez. & $\begin{array}{c}8,53 \\
(8,13-8,94)\end{array}$ & $\begin{array}{c}8,61 \\
(8,24-8,98)\end{array}$ \\
\hline BD4. Utilizan un lenguaje claro en los formularios. & $\begin{array}{c}7,78 \\
7,72 \\
(7,21-8,35)\end{array}$ & $\begin{array}{c}7,24-8,19) \\
8,43\end{array}$ \\
\hline BD5. Cuentan con un personal preparado. & $(7,30-8,34)$ & $(8,04-8,83)$ \\
\hline BD10. El personal le ha informado y realizado los trámites & $\begin{array}{c}8,13 \\
\text { sin errores. }\end{array}$ & $(7,49-8,78)$ \\
\hline BD13. Si retiro documentación el personal ha realizado un \\
seguimiento razonado.
\end{tabular}

(IC) Intervalo de confianza al 95,0\% de probabilidad.

Escala de 1 a 10 siendo el 1 la opinión más desfavorable y el 10 la más favorable. 
En cuanto a las características con las que se realiza el servicio de búsqueda de documentos, los usuarios del archivo muestran una opinión muy favorable en cuanto al horario, atención facilitada por teléfono, correo electrónico o fax, Esta opinión es más crítica ante aspectos tales como las condiciones del emplazamiento del archivo, la señalización, o el entorno en el que se realiza el servicio. Como se puede observar en la Tabla VII, esta opinión no se modifica tras la certificación del sistema de gestión de la calidad.

\section{TABLA VII}

Opinión de los usuarios del AGUCM sobre las condiciones de acceso y el entorno en el que se realiza el proceso de búsquedas documentales

\begin{tabular}{|c|c|c|}
\hline Preguntas & $\begin{array}{l}\text { Años 2002-2004 } \\
\text { Valor medio (IC) }\end{array}$ & $\begin{array}{l}\text { Años 2006-2008 } \\
\text { Valor medio (IC) }\end{array}$ \\
\hline BD6. El horario del servicio es suficiente. & $\begin{array}{c}7,85 \\
(7,31-8,39)\end{array}$ & $\begin{array}{c}8,25 \\
(7,91-8,58)\end{array}$ \\
\hline BD7. El acceso tiene una señalización adecuada. & $\begin{array}{c}6,33 \\
(5,64-7,03)\end{array}$ & $\begin{array}{c}7,06 \\
(6,50-7,61)\end{array}$ \\
\hline BD8. El emplazamiento del servicio es el adecuado. & $\begin{array}{c}6,20 \\
(5,54-6,86)\end{array}$ & $\begin{array}{c}6,70 \\
(6,13-7,28)\end{array}$ \\
\hline BD9. El entorno de atención al público es el adecuado. & $\begin{array}{c}5,84 \\
(5,01-6,66)\end{array}$ & $\begin{array}{c}6,412 \\
(5,79-7,04)\end{array}$ \\
\hline $\begin{array}{l}\text { BD11. Se le facilita atención por teléfono, fax, o correo } \\
\text { electrónico. }\end{array}$ & $\begin{array}{c}7,91 \\
(7,21-8,61)\end{array}$ & $\begin{array}{c}8,518 \\
(8,09-8,94)\end{array}$ \\
\hline $\begin{array}{l}\text { BD12. No ha sufrido interrupciones mientras ha sido aten- } \\
\text { dido. }\end{array}$ & $\begin{array}{c}7,95 \\
(7,38-8,52)\end{array}$ & $\begin{array}{c}8,30 \\
(7,90-8,70)\end{array}$ \\
\hline BD14. De una valoración global de la calidad del servicio. & $\begin{array}{c}7,89 \\
(7,42-8,36)\end{array}$ & $\begin{array}{c}7,91 \\
(7,49-8,33)\end{array}$ \\
\hline
\end{tabular}

(IC) Intervalo de confianza al 95,0\% de probabilidad.

Escala de 1 a 10 siendo el 1 la opinión más desfavorable y el 10 la más favorable.

\section{Discusión y conclusiones}

El espectacular desarrollo en los últimos años de la certificación de los sistemas de gestión de la calidad siguiendo la Norma Internacional ISO 9001:2000 en el sector empresarial privado, ha venido determinado tanto por factores internos como externos a la organización. De entre los factores externos, el más importante es el de ser un requisito imprescindible en muchas empresas para poder comercializar sus productos o servicios; entre los factores internos, los elementos que más motivan a las organizaciones se refieren a los beneficios potenciales que dicho sistema puede tener sobre la mejora de la gestión en general, la consecu- 
ción de un mejor control de las operaciones que se realizan, o un mejor control de la documentación, así como la mejora de la calidad de los productos y del servicio al cliente, e incluso la consecución de una mejora de la productividad en general (Heras, 2006).

En contraste con lo indicado más arriba, las motivaciones que han llevado a los gestores de los servicios de información y documentación a implantar sistemas de gestión de la calidad son, entre otras, la consecución de la mejora en la satisfacción de los usuarios y la mejora en la documentación y en las operaciones de los procesos (Kostagiolas y Kitsiou, 2008) o, como refiere Balagué Mola (2007), el de conseguir una mejora de la calidad de los servicios y el de alinearse con la estrategia de calidad de la universidad, en el caso de las bibliotecas universitarias.

En el caso del AGUCM, la implantación de un sistema de gestión de la calidad, y su posterior certificación, vino determinada por la combinación de dos factores: el primero, y más importante, el de ser un objetivo que se planteó la dirección del archivo tras el proceso de evaluación llevado a cabo en el año 2001, con la finalidad de mejorar el desempeño y el control de los procesos de trabajo; a este objetivo se le sumó como un segundo factor el hecho de que en la estrategia para la calidad dentro de la UCM se potenciaron, en ese momento, los procesos de certificación de los servicios y unidades administrativas.

Los resultados presentados en este trabajo tienen la singularidad, con respecto a los referidos en la literatura (ver para una reciente revisión, Sampaio, 2009), el de ser el único caso en donde se disponen de datos de opinión de los clientes y del personal antes y después de la certificación de su sistema de gestión de la calidad.

De acuerdo con los datos obtenidos de la opinión del personal del archivo, los beneficios más sobresalientes se consiguen en aquellas áreas que dependen en exclusiva del trabajo y de la conducta del propio personal. La apreciación sobre la calidad de los recursos no se modifica por la certificación, sin embargo, se mejoran los aspectos relacionados con la gestión de los procesos y con la conducta del personal, el cual muestra un mayor sentimiento de pertenencia a la organización, una mayor participación en el establecimiento de objetivos y se muestra más activo a la hora de potenciar el trabajo en grupo. La satisfacción del personal con el trabajo no se ve modificada, posiblemente debido a la valoración que ya poseía (un 7,7 frente a un 8,4).

Con respecto a los resultados referidos a la opinión de los usuarios del archivo, se puede concluir con que la certificación no produce ninguna mejora en la percepción de éstos sobre los servicios prestados por el archivo. No obstante, debemos indicar que las valoraciones que hacen los usuarios de todos aquellos aspectos relacionados con la calidad del servicio que se les presta, se mantienen muy altas antes y después de la certificación, hecho que se debe, posiblemente, a las acciones de mejora realizadas después del proceso de evaluación llevado a cabo en el año 2001.

En los estudios realizados en el sector industrial o de servicios, los datos sobre los beneficios internos que se consiguen por la certificación son contradic- 
torios debido, en parte, a que la percepción de estos beneficios viene determinada por la importancia que tiene para los empleados la naturaleza e intensidad de las motivaciones para conseguir la certificación (Boiral y Roy, 2007; Sampaio, 2009).

Como ejemplo, podemos citar los estudios que encuentran que la certificación tiene poco impacto sobre la actitud y la conducta de los empleados (Van der Wiele, 2005), o sobre su satisfacción (Brown, 1998; Douglas, 2003); sin embargo, Casadesus y Karapetrovic (2005) obtienen, en una muestra de 339 compañías catalanas, un impacto positivo sobre la satisfacción de los empleados, y la salud y la seguridad en el trabajo (60\%). No obstante, sólo el 50\% de las compañías indican una mejora en la participación del personal en el trabajo en grupo y únicamente el $40 \%$ señalan que se incrementa la satisfacción en el trabajo.

Con respecto a la mejora en el control de la gestión, y en el desempeño o eficiencia de los procesos, los datos son también contradictorios. Douglas (2003), en un estudio sobre 104 compañías del Reino Unido, indican que son mayoría los gestores que señalan que no existe una mejora; sin embargo, Feng y otros (2008) en una muestra realizada en 613 compañías de Australia y Nueva Zelanda, encuentran una relación positiva entre la certificación y la mejora de las actividades operativas.

En el caso de las bibliotecas, Kostagiolas y Kitsiou (2008) indican que, en opinión de los directores de las bibliotecas, durante el proceso de implementación del sistema de gestión de la calidad se mejoró la asunción de las nuevas responsabilidades, la provisión de instrucciones, se aislaron las ineficiencias y se pudieron introducir mejoras en el funcionamiento. Balagué Mola (2007) en su estudio sobre 121 bibliotecas universitarias, señala que entre los beneficios que se obtienen con la certificación están: el de promover la mejora continua, el de la toma de decisiones basadas en datos, el de la definición de las responsabilidades, el de estandarización de los procesos de trabajo y el de documentación de los procedimientos. Otros beneficios, con un menor nivel de consenso, son: la mejora de la competencia de los recursos humanos, la mejora de la moral del personal, o el reconocimiento profesional.

\subsection{Conclusiones}

De los resultados que se han obtenido en el estudio del caso del Archivo General de la Universidad Complutense, podemos establecer las siguientes conclusiones:

1. Reconocer la importancia que han tenido los diferentes planes institucionales de evaluación de la calidad, establecidos por el Consejo de Universidades, en la introducción y desarrollo de una cultura sobre la calidad. Un caso particular del impacto de estos planes han sido las mejoras establecidas en los servicios de información y de documentación de las 
universidades españolas y el desarrollo de sistemas de gestión de la calidad, como el aquí presentado.

2. La importancia que ha tenido para la implantación del sistema de gestión de la calidad la presencia de un fuerte liderazgo en la dirección del archivo, y de un personal altamente motivado que participó plenamente en el proyecto desde su inicio. A esta fortaleza, habría que añadir el apoyo institucional de la universidad, imprescindible para la culminación del proyecto.

3. La implantación de un sistema de gestión de la calidad siguiendo los requisitos de la Norma Internacional ISO 9001:2000 tiene los siguientes beneficios:

- Aportar un mayor rigor a la gestión de la unidad, debido a que los requisitos de la norma ISO 9001:2000 exige el establecimiento de una política de la calidad, la definición de objetivos, el establecimiento de canales de comunicación interna y una mayor claridad y transparencia en la distribución de responsabilidades entre el personal. Como ejemplo, el AGUCM, tiene definido un organigrama y, para cada uno de los puestos de trabajo, tiene establecido el perfil del puesto, sus responsabilidades, y competencias.

- Una mejora evidente en el desempeño de los procesos operativos, mediante la mejora de la documentación de cada uno de éstos, el establecimiento de indicadores, su medición y seguimiento, y el dotar al personal del necesario enfoque a procesos para la mejora de su gestión y de los resultados que se desean conseguir.

- Un incremento en la asunción de responsabilidades por parte del personal, y en su sentido de pertenencia a una organización, ya que todos comparten unos objetivos comunes.

4. La obligación de mantener un compromiso continuado con la mejora del sistema de gestión de la calidad implantado, debido a que el archivo tiene que someterse periódicamente a procesos de auditoría externa para renovar su certificación.

\section{Referencias}

Balagué Mola, N. (2007): Les normes de qualitat ISO 9000 a les biblioteques d'institucions d'educació superior. Universitat de Barcelona. http://www.tdx.cesca.es/TESIS_UB/ AVAILABLE/TDX-0423107-123746//NBM_TESI.pdf [consulta: 14 de enero de 2009].

Boiral, O., y Roy, M. J. (2007): ISO 9000: integration rationales and organizational impacts. International Journal of Operations \& Production Management, vol. 27 (2), 226-247.

Brophy, P. (1994): The quality program of the library and learning resources service at the University of Central Lancashire. Inspel, vol. 28 (2), 240-247. Disponible en http:// forge.fh-potsdam.de/ IFLA/INSPEL/94-2brpe.pdf [consulta: 9 de febrero de 2009]. 
Brown, A.; Wiele, T. V., y Loughton, K. (1998): Smaller enterprises experiences with ISO 9000. The International Journal of Quality \& Reliability Management, vol. 15 (3), 273-285.

Brumm, E. K. (1996): The marriage of quality standards and records management. Records Management Quarterly, vol. 30 (2), 3-11.

Casadesus, M., y Karapetrovic, S. (2005): The erosion of ISO 9000 benefits: a temporal study. The International Journal of Quality \& Reliability Management, vol. 22 (2/3), 120- 136.

Consejo de Universidades. (2000): Guía de Evaluación de Servicios. Madrid, Ministerio de Educación, Cultura y Deporte. Secretaria General Técnica.

Cruellas i Serra, R. M, y Petit i Cibiriain, C. (2005): La implantació d'un sistema de gestió de la qualitat a l'Arxiu Nacional de Catalunya, d'accord amb la Norma ISO 9001:2000. Lligal. Revista Catalana d'arxivistica, vol. 24, 321-366.

Douglas, A.; Coleman, S., y Oddy, R. (2003): The case for ISO 9000. The TQM Magazine, vol. 15 (5), 316-324.

Duff, W., y Mckemmish, S. (2000): Metadata and ISO 9000 compliance. Information Management Journal, vol. 34 (1), 4-16.

Falomir, V., y Segarra, J. R. (2006): El Sistema de gestión de la Calidad (Norma ISO 9001:2000) en la Biblioteca de la Universitat Jaume I de Castellón. Boletín ANABAD, vol. 56 (1), 75-87.

Feng, M.; Terziovsky, M., y Samson, D. (2008): Relationship of ISO 9001:2000 quality system certification with operational and business performance: a survey in Australia and New Zealand based manufacturing and service companies. Journal of Manufacturing Technology Management, vol. 19 (1), 22-37.

Flores Varela, C. (2003): El Archivo General de la Universidad Complutense: punto de inflexión. Archivos universitarios e historia de las universidades, Getafe (Madrid), Dykinson, 131-152. Disponible en http://eprints.ucm.es/8587/ [consultado el 15 de diciembre de 2008].

Flores Varela, C. (2008): El Archivo General de la Universidad Complutense de Madrid y la gestión de la calidad. Boletín ANABAD, vol. 68 (1), 127-133.

Grabolosa Sellabona, M., y Vicens Perpinya, J. (2000): Funcionament d'un centre de documentació amb la ISO 9002: el cas del Centre de Documentació del Parc Natural de la Zona Volcànica de la Grarrotxa. Biblioteca Informacions, Barcelona, vol. 22, 14-18.

Gunnlaugsdottir, J. (2002): The quality must be on record: a survey of organisations having an ISO 9000 certification in Iceland. Records Management Journal, 12 (2), 40-47.

Heras Saizarbitoria, I.; Arana Landin, G., y Casadesus, M. A. (2006): Delphi study for ISO 9000 and EFQM. International Journal of Quality \& Reliability Management, vol. 23 (7), 807-827.

Houten, Gv. (1996): The ISO document tidal wave. Records Management Quarterly, vol. 30 (2), 12-20.

Houten, Gv. (2000): ISO 9001:2000. Information Management Journal, vol. 34 (2), 28-37.

Krestel, H. (2006): Certification of a patent information centre according to DIN EN ISO 9001. World Patent Information, vol. 22, 19-22.

Kostagiolas, P., y Kitsiou, M. B. (2008): Issues and perceptions for ISO 9000 implementa- 
tion in Greek academic libraries. Library Management, vol. 29 (6/7), 583-599.

Lannon, K.M. (1995): ISO 9000 records requirements: information management impact. FID News Bulletin, vol. 45 (6), 181-194.

Martínez Micó, M. A. (2001): El Departamento de Gestión Documental de la Diputación de Alicante, un Departamento que trabaja en calidad. Revista d'Arxius $i$ Biblioteques COMPACTUS, vol. 2 (set-desem), 20-23.

McClave, J. T; Benson, P. G.; Sincich, T. (1998): Statistics for business and economics. (7. ${ }^{\mathrm{a}}$ ed.), EEUU: Prentice Hall Inc.

Ministerio de Educación Cultura y Deporte. Real Decreto 408/2001, de 20 de abril, por el que se establece el II Plan de la Calidad de las Universidades.

Murillo, I., y López de Castro, S. (2002): La calidad en el Archivo Municipal de Logroño. Introducción al sistema. Disponible en http://www.logro-o.org/documentos/200708011259_1-\%20INTRODUCCION\%20AL\%20SISTEMA\%20DE\%20CALIDAD.pdf [consultado el 16 de septiembre de 2008].

Núñez Fernández, E. (2007): Archivos y Normas ISO. Madrid: Trea.

Real, M. L. (2006): A gestão do Arquivo Municipal do Porto: experiência inovadora para o desenvolvimento de um Sistema de Gestão da Qualidade, em Arquivos, nos termos da Norma NP-EN ISSO 9001:2000. Paginas Arquivos \& Bibliotecas A \& B, vol. 18, 7-70.

Sampaio, P.; Saraiva, P., y Rodrigues, A. G. (2009): ISO 9001 certification research: questions, answers and approaches. The International Journal of Quality $\&$ Reliability, vol. 26 (1), 38-58

Sebina, P. M. (2001): ISO standards and records management: the case of Botswana Meat Commission. African Journal of Library, Archives E Information Science, 11 (1), 49-59.

Stephens, D. O. (1996): ISO 9000 and international records management. Records Management Quarterly, vol. 30 (3), 67-73.

The ISO Survey 2007. Switzerland, ISO Central Secretariat, 2008.

Unidad de Sistemas de Información del Ayuntamiento de Zaragoza. Disponible en www. zaragoza.es/ciudad/usic/calidad/iso.htm [consultado el 18/02/2009].

UNE-EN-ISO 9000: 2000. Sistemas de gestión de la calidad. Requisitos. Madrid: Aenor 2000.

Valls, V. M., y Vergueiro, W. C. S. (2006): Quality management on information services according to ISO 9000. New Library World, vol. 107 (1231/1232), 523-537.

Vasseur, M. C. (1998): A coeur de la demarche du certification ISO 9000: Le Centre de Documentation de la Societé Grenobloise d'Etudes et d'Applications Hydrauliques, Bulletin des Bibliotheques de France, vol. 44(1), 44-46.

Weise, C. E., y Stamoolis, P. G. (1993): ISO 9000: an opportunity for records management professionals. Records Management Quarterly, vol. 27(4), 3-11.

Wiele, T. V.; Iwarden, J. V., y Williams, R. (2005): Perceptions about the ISO 9000 (2000) quality system standard revision and its value: the Dutch experience. The International Journal of Quality \& Reliability, vol. 22, (2), 101-119.

Yusof, Z. M., y Chell, R. W. (1998): How records management could help Malaysia materialize its Vision 2020. Journal of Librarianship and Information Science, vol. 30 (3), $169-175$. 